\title{
GENERIC PARAMETERS GOVERNING THE BIOFUNCTIONALITY OF LASER SURFACE ENGINEERED NYLON 6,6
}

\author{
Paper \#1204
}

David G. Waugh and Jonathan Lawrence

School of Engineering, University of Lincoln, Brayford Pool, Lincoln, Lincolnshire, LN6 7TS, UK

\begin{abstract}
On account of an ever increasing demand on medicine there is a need and a drive by the biomedical industry to develop robust and predictable implant technology. This paper gives an account of the implementation of $\mathrm{CO}_{2}$ and $\mathrm{KrF}$ excimer laser systems to modulate the biofunctionality of nylon 6,6 in terms of osteoblast cell response. There were correlative trends between the cell response, contact angle, polar component and surface oxygen content for the whole area irradiative processed samples. Thus, allowing one to identify the potential for this technology in regenerative medicine. However, no strong correlations were determined for the laser-induced patterned samples which can be attributed to the likely mixed-state wetting regime. Through analytical analysis, governing equations are discussed, showing how different parameters can be used to predict the wettability of, and biological cell response to, laser surface engineered nylon 6,6.
\end{abstract}

\section{Introduction}

On account of people living longer, a number of institutions have developed a focus on bioengineering to meet the ever growing demands on medical facilities [1]. As a result, these needs have to be met more economically and efficiently so that costs and the need for unnecessary surgery are considerably reduced. Therefore, it is imperative for the biomedical industry to devise a way to manufacture cheap implants which can be used in confidence to ensure a dramatic reduction in failure rates.

Biotechnology can be seen to improve upon quality of life and therefore is a vital necessity to significantly advance the general health of society, especially when we take in to account the aging population who are being required to work longer in their lifetime. On account of the success of this industry many have identified that further growth will arise from the exploitation of inexpensive and relatively easier to manufacture polymeric biomaterials. Even though the use of polymeric materials in the biotechnology industry is becoming a rapidly developing area of research it has been found in a number of instances that the performance of these materials are not sufficient and can lead to insufficient bioactivity [2].

The control of cell adhesion to synthetic polymers is a key factor in tissue engineering, resting on the ability to direct specific cell types to proliferate, migrate and express physiological behaviours in order to support and stimulate cellular architecture and tissue reconstruction [3]. A considerable amount of research effort has therefore been devoted to the surface modification of materials to enhance cell adhesion and growth $[4,5]$. Laser surface modification provides a means of not only modifying the surface of a polymer and leaving the bulk virtually unchanged, but can also generate discrete and specific changes to the surface properties of polymers.

Even though it is recognized that the roughness, rigidity, charge and chemistry of the surface of a polymer play an important role in influencing cell behaviour; the wettability characteristics of polymers has received considerable attention on account of the fact that wetting is often the primary factor dictating whether a liquid film or a coating will initially adhere and then bond to a substrate. As a result, several research groups have studied the interactions of different types of cultured cells with various polymers and copolymers with different wettabilities to correlate the relationship between surface wettability and blood-, cell-, or tissue-compatibility [6-8]. One problem seen from studies using different kinds of polymer is that the surfaces are heterogeneous both chemically and physically (different surface chemistry, roughness, rigidity, crystallinity, and so on), which may result in considerable variation. To overcome this variation issue, numerous techniques for surface treatment for altering the wettability characteristics of polymers have been investigated [9-12]. But none of these techniques offer the controllability, precision, selectivity and flexibility of laser processing - both infrared (IR) and ultraviolet (UV) $[2,13]$.

Built upon previous research $[14,15]$ this paper details the study of osteoblast cell growth on laser surface treated nylon 6,6 and discusses potential governing 
trends and equations to predict and quantify the cell response.

\section{Experimental Technique}

\section{Nylon 6,6 Material}

The nylon 6,6 $\left(\mathrm{T}_{\mathrm{m}}: 255^{\circ}, \rho: 1.3 \mathrm{gcm}^{-3}\right)$ was sourced in $100 \mathrm{~mm}^{2}$ sheets with a thickness of $5 \mathrm{~mm}$ (Goodfellow Cambridge, Ltd). To obtain a conveniently sized sample for experimentation the as-received nylon sheet was cut into $20 \mathrm{~mm}$ diameter discs using a $1 \mathrm{~kW}$ continuous wave (cw) $\mathrm{CO}_{2}$ laser (Everlase S48; Coherent, Ltd).

\section{$\mathrm{CO}_{2}$ Laser-Induced Patterning}

There were four patterns induced onto the surfaces of the nylon 6,6 samples; these were trenches with $50 \mu \mathrm{m}$ spacing (CT50), hatches with $50 \mu \mathrm{m}$ spacing (CH50), trenches with $100 \mu \mathrm{m}$ spacing (CT50) and hatches with $100 \mu \mathrm{m}$ spacing (CH100). In addition, an as-received control sample was used (AR). For each of the irradiated patterns the laser power was set to $70 \%$ (7 W) operating at $600 \mathrm{mms}^{-1}$.

Further details of the $\mathrm{CO}_{2}$ laser-induced patterning can be found in [16].

\section{$\mathrm{CO}_{2}$ Laser Whole Area Irradiative Processing}

A cw $100 \mathrm{~W} \mathrm{CO}_{2}$ laser (DLC; Spectron, Ltd) was used to scan a $5 \mathrm{~mm}$ diameter beam across the target sample with one pass in order to irradiate the test area with an irradiance of $510 \mathrm{Wcm}^{2}$. By using a galvanometer, scanning speeds of $150,100,75,50,25$ and $20 \mathrm{mms}^{-1}$ were employed to irradiate six samples with effective fluences of 16.84 (samples CWA17), 25.51 (sample CWA26), 34.18 ( sample CWA34), 51.02 (sample CWA51), 102.04 (sample CWA102) and 127.55 (sample CWA128) $\mathrm{Jcm}^{-2}$, respectively. As with the laser-induced patterning experimentation the samples were held in place on a $\mathrm{z}$-variable stage with the whole experiment being carried out in a laser safety cabinet with the ambient gas being air.

\section{KrF Excimer Laser-Induced Patterning}

Two non-contact masks were used for both dimensioned patterns which included a brass mask with six $1 \mathrm{~mm}$ diameter holes spaced by $2 \mathrm{~mm}$, centre to centre, for the $100 \mu \mathrm{m}$ dimensions and a SS316 foil (Laser Micromachining Ltd., UK) with five $0.5 \mathrm{~mm}$ diameter holes spaced by $1.5 \mathrm{~mm}$, 'centre to centre', for the $50 \mu \mathrm{m}$ dimensions. To keep a constant 10 pulses per site it should also be noted here that scanning velocities of $0.125 \mathrm{mms}^{-1}$ and $0.25 \mathrm{mms}^{-1}$ were used for the $50 \mu \mathrm{m}$ and $100 \mu \mathrm{m}$ dimensioned patterns, respectively.

Further details of the $\mathrm{KrF}$ excimer laser-induced patterning can be found in [17].

\section{KrF Excimer Laser Whole Area Irradiative Processing}

For the large area processing experiments 6 samples were studied; these being 100 pulses at $100 \mathrm{~mJ}$ (EWA100), 100 pulses at $150 \mathrm{~mJ}$ (EWA150), 100 pulses at $200 \mathrm{~mJ}$ (EWA200), 100 pulses at $250 \mathrm{~mJ}$ (EWA250), 500 pulses at $250 \mathrm{~mJ}$ (EWA250_500) and 1000 pulses at $250 \mathrm{~mJ}$ (EWA250_1000). This gave fluences of $36 \pm 3,54 \pm 5,72 \pm 8$ and $91 \pm 10 \mathrm{mJcm}^{-2}$, respectively for the different energies used.

Further details of the $\mathrm{KrF}$ excimer laser whole area irradiative processing can be found in [17].

\section{Topography, Wettability Characteristics and Surface Chemistry Analysis}

After laser irradiation the nylon 6,6 samples were analysed using a number of techniques. The surface profiles were determined using a white light interferometer (WLI) (NewView 500; Zygo, Ltd) with MetroPro and TalyMap Gold Software. The WLI was set-up using a $\times 10$ Mirau lens with a zoom of $\times 0.5$ and working distance of $7.6 \mathrm{~mm}$. This system also allowed $\mathrm{Sa}$ and $\mathrm{Ra}$ roughness parameters to be determined for each sample.

The samples were ultrasonically cleaned in isoproponal (Fisher Scientific Ltd.) for 3 minutes at room temperature before using a sessile drop device to determine various wettability characteristics. This was to allow for a relatively clean surface prior to any contact angle $(\theta)$ measurements being taken. To ensure that the sample surfaces were dry a specimen dryer (Metaserv, Ltd.) was employed to blow ambient air across the samples. A sessile drop device (OCA20; Dataphysics Instruments, $\mathrm{GmbH}$ ) was used with relevant software (SCA20; Dataphysics Intrsuments, $\mathrm{GmbH}$ ) to allow the recent advancing $\theta$ for triply distilled water and the recent advancing angle for diiodomethane to be determined for each sample. Thereafter the advancing $\theta$ for the two liquids were used by the software to draw an OWRK plot to determine the surface energy of the samples. For the two reference liquids the SCA20 software used the Ström et al technique (triply distilled water SFT(total:72.80), $\quad$ SFT(D:21.80), $\quad$ SFT(P:51.00); diiodomethane - SFT(total:50.80), SFT(D:50.80), SFT(P:0.00)) to calculate the surface energy of the material. It should be noted here that ten $\theta$, using two 
droplets in each instance, were recorded to achieve a mean $\theta$ for each liquid and surface.

All samples were analysed using x-ray photoelectron spectroscopy (XPS). This allowed any surface modifications in terms of surface oxygen content due to the laser irradiation to be revealed. Details of the set-up and application of the XPS analysis can be found in [14].

\section{In Vitro Experimentation}

Prior to any biological testing being carried out the samples were autoclaved (D-Series Bench-Top Autoclave; Systec, $\mathrm{GmbH}$ ) to ensure that all samples were sterilized. For all biological work undertaken, unless stated, a biological safety cabinet (BSC) (Microflow Class II ABS Cabinet; BioQuell UK, Ltd) was used to create a safe working environment and to provide a clean, sterile environment to manipulate the cells used.

Normal human osteoblast cells (Clonetics CC-2538; Lonza, Inc.) were initially cultured in a T75 $(75 \mathrm{ml})$ flask by suspending the cells in $19 \mathrm{ml}$ culture medium comprising of $90 \%$ eagle minimum essential medium (Sigma-Aldrich, Ltd., UK) and $10 \%$ foetal bovine serum (FBS) (Sigma-Aldrich Ltd., UK). The flask was then placed in an incubator and left for $24 \mathrm{hrs}$. After $24 \mathrm{hrs}$ the cells were assessed and the spent media was aspirated before dispensing $15 \mathrm{ml}$ of fresh media and returning the flask to the incubator for three days.

The period of three days allowed the cells to become confluent in the flask providing enough cells for seeding onto the samples. The cells were detached from the flask using $5 \mathrm{ml}$ Trypsin-EDTA (SigmaAldrich Ltd., UK) whilst placed in the incubator for seven minutes. Once all cells had become detached $10 \mathrm{ml}$ culture medium was added to neutralize the Trypsin. In order to aspirate the supernatant the cell culture was centrifuged (U-320R; Boeco, $\mathrm{GmbH}$ ) for five minutes at $200 \mathrm{~g}$. To ensure the cells were ready for seeding they were re-suspended in $10 \mathrm{ml}$ of culture medium and dispensed between the samples in 6-well plates. This equated to $0.55 \mathrm{ml}\left(2 \times 10^{4}\right.$ cells $\left./ \mathrm{ml}\right)$ for each sample. The well plates were then placed in the incubator for a set time. One plate was removed after $24 \mathrm{hrs}$ and two other plates after four days. A well plate after $24 \mathrm{hrs}$ and 4 days was prepared for the SEM as will be discussed later and the other plate, removed after 4 days, was prepared for counting using an improved neubauer hemacytometer (Fisher Scientific Ltd., UK) by mixing $10 \mu \mathrm{l}$ of each cell suspension with $10 \mu \mathrm{l}$ of trypan-blue (Sigma-Aldrich Ltd., UK). In order to harvest the cells for counting the cells were detached from the samples using $2 \mathrm{ml}$ Trypsin-EDTA
(Sigma-Aldrich Ltd., UK) whilst placed in the incubator for seven minutes. Once all cells had become detached $4 \mathrm{ml}$ culture medium was added to neutralize the Trypsin. In order to aspirate the supernatant the cell culture was centrifuged (U-320R; Boeco, $\mathrm{GmbH}$ ) for five minutes at $200 \mathrm{~g}$. To ensure the cells were ready for counting they were resuspended in $2 \mathrm{ml}$ of culture medium and $2 \mathrm{ml}$ of the trypan-blue was added.

\section{Scanning Electron Microscopy and Cell Cover Density of In Vitro Samples}

In order to view the attached cells using SEM it was necessary to undertake a procedure to produce a sample that was dehydrated ready for Au coating. The full detailed procedure can be found in [15].

The cell cover density was determined following both $24 \mathrm{hrs}$ and 4 day incubation. This was done by analysing the cell coverage on each sample using SEM and optical micrographs with the ImagePro software. The optical micrographs were obtained using an upright optical microscope (Flash 200 Smartscope; OGP, Ltd) with objective magnifications varying between $\times 10$ and $\times 100$.

\section{Analytical Analysis for Governing Equations}

To determine potential governing equations the data was analyzed using genetic programming and symbolic regression software (Eureqa; Cornell Creative Machines Laboratory, USA) [18]. It should be noted here that all data was normalized prior to analysis.

\section{Results and Discussion}

\section{Wettability \\ $\underline{\mathrm{CO}}_{2}$ and $\mathrm{KrF}$ Excimer Laser-Induced Patterning}

Throughout this work it was observed that $\mathrm{CO}_{2}, \mathrm{KrF}$ excimer laser-induced patterning of nylon 6,6 inherently gave rise to a significant increase in $\theta$. This could be seen to be on account of the likelihood of a mixed-state wetting regime taking place along the liquid-surface interface [19].

Current theory has not been corroborated with, in terms of the laser-induced patterning of nylon 6,6, insofar as the increase in $\mathrm{Sa}$ and $\mathrm{Ra}$ did not give rise to a reduction in $\theta$. However, it has been seen that surface energy parameters may have a significant impact upon the wetting nature of nylon 6,6 even if a wetting transition has taken place. Figure 1 and Figure 2 show the relationship between $\theta$ and $\gamma^{\mathrm{P}}$ and $\gamma^{\mathrm{T}}$, respectively. 
From these graphs it is possible to identify that by collating all results for the patterning of nylon $6,6 \theta$ was a strong inverse function in correlation with $\gamma^{\mathrm{P}}$ and $\gamma^{\mathrm{T}}$. On account of this, it is reasonable to say that for the patterned nylon 6,6 samples $\gamma^{\mathrm{P}}$ and $\gamma^{\mathrm{T}}$ were dominant parameters for determining the wettability.

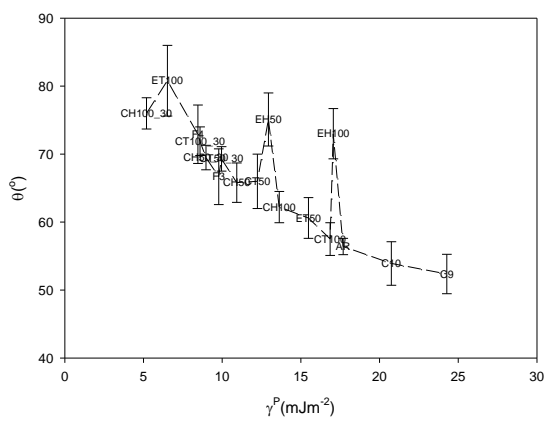

Figure 1 - Graph showing the correlation between $\theta$ and $\gamma^{\mathrm{P}}$.

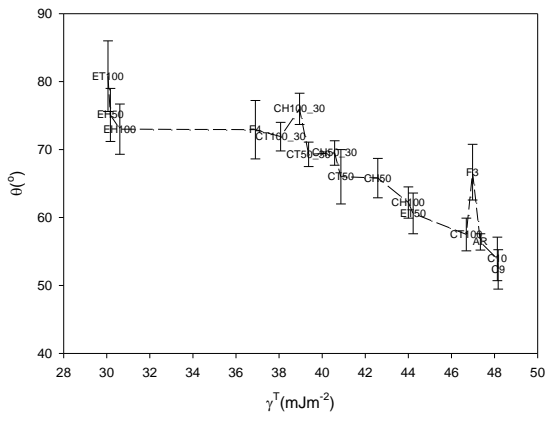

Figure 2 - Graph showing the correlation between $\theta$ and $\gamma^{\mathrm{T}}$.

It was seen that the surface roughness in terms of $\mathrm{Ra}$ and $\mathrm{Sa}$ did not seem to have a dominant role in determining $\theta$ for the laser-induced patterned nylon 6,6 samples. This was due to $\theta$ being slightly erratic as the surface roughness increased.

$\mathrm{CO}_{2}$ and $\mathrm{KrF}$ Excimer Laser Whole Area Irradiative Processing

The work involving $\mathrm{CO}_{2}$ and $\mathrm{KrF}$ excimer laser whole area irradiative processing showed that the wettability characteristics of nylon 6,6 did tend to correspond with current theory in that $\theta$ decreased on account of an increase in surface roughness, increase in $\gamma^{\mathrm{P}}$, increase in $\gamma^{\mathrm{T}}$ and increase in surface oxygen content. From Figure 3 it was found, upon collating the results for all laser whole area irradiative processed nylon 6,6 samples, that there was a strong inverse relationship between $\theta$ and $\gamma^{\mathrm{P}}$. In addition to this, it can also be seen from Figure 4 that $\gamma^{\mathrm{T}}$ showed a similar correlation with $\theta$. This further suggests that the surface energy parameters are the most dominant parameter in giving rise to $\theta$ for laser-treated nylon 6,6 . What is more, Figure 3 and Figure 4 indicate that it may be possible to use $\gamma^{\mathrm{P}}$ and $\gamma^{\mathrm{T}}$ to predict $\theta$ on account of the fact that they show that the results are reproducible as samples which had similar values of either $\gamma^{\mathrm{P}}$ and $\gamma^{\mathrm{T}}$ gave rise to equivalent $\theta$.

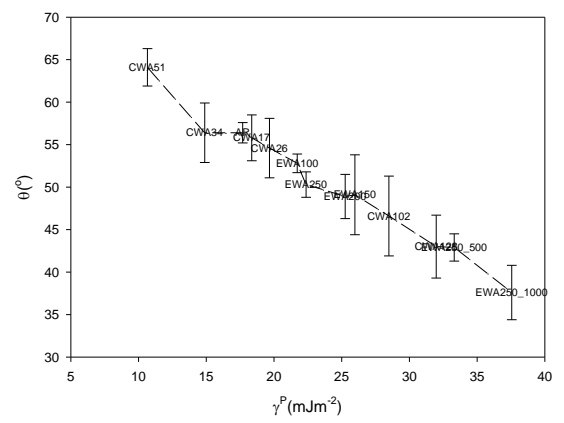

Figure 3 - Graph showing the correlation between $\theta$ and $\gamma^{\mathrm{P}}$.

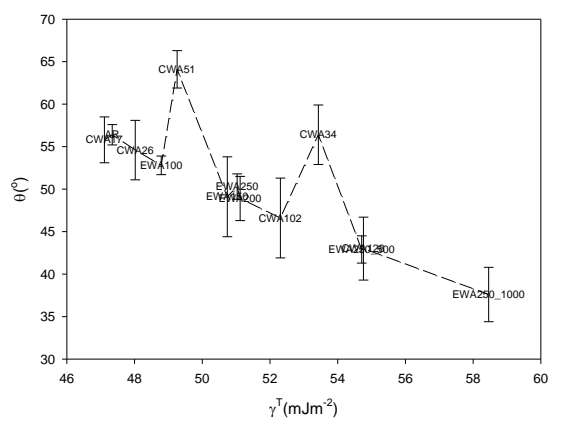

Figure 4 - Graph showing the correlation between $\theta$ and $\gamma^{T}$.

As surface roughness had increased, it was believed that $\mathrm{Sa}$ and $\mathrm{Ra}$ would have a significant impact upon $\theta$; however, the surface roughness did not tend to have any particular correlation with $\theta$.

Comparisons Between Laser-Induced Patterning and Laser Whole Area Irradiative Processing

By taking into account all results that have been obtained it was possible to ascertain the effect of all surface parameters studied on $\theta$. For all $\mathrm{CO}_{2}$ and $\mathrm{KrF}$ excimer laser-induced patterning of nylon 6,6 the surface oxygen content had increased which can be attributed to oxidation of the surface during the laser processing. It is reasonable to conclude from the results obtained that surface oxygen content in this instance was not a main driving parameter which accounts for the measured $\theta$. This is owed to variations 
in $\theta$ between the laser-induced patterned and laser whole area irradiative processed samples even though, in all instances, the surface oxygen content increased.

When compared to one another it was found that all laser-induced surface patterning elicited an increase in $\theta$; whereas the laser whole area irradiative processing of the nylon 6,6 gave rise to a more hydrophilic surface in which $\theta$ decreased. In order to explain the increase in $\theta$ for the laser-induced patterned samples a mixedstate wetting regime is proposed in which both CassieBaxter and Wenzel regimes occur along the interface once the droplet is in equilibrium. This mixed-state wetting regime is believed to arise from the rough periodic patterns induced by the laser processing, allowing one to infer that the periodic topographical patterns have a dominant role in the determination of $\theta$.

\section{Cell Morphology}

\section{Hour Incubation}

For all samples the osteoblast cells, to some extent, adhered to the differing surfaces. That is, the cells firstly attached, adhered and began to spread across the surfaces. The extent to which this phenomenon took place appeared to be dependent on the laser processing. In terms of cell morphology it can be seen that following $24 \mathrm{hrs}$ of incubation the osteoblast cells on both the as-received and most of the laser surface treated samples were bipolar in nature. This is evidenced in Figure 5 and Figure 6. This suggests that after $24 \mathrm{hrs}$ of cell adhesion and growth there is no difference in terms of cell signaling for most of the samples. Having said that, some differences did arise on some of the laser surface treated samples and these will now be discussed.

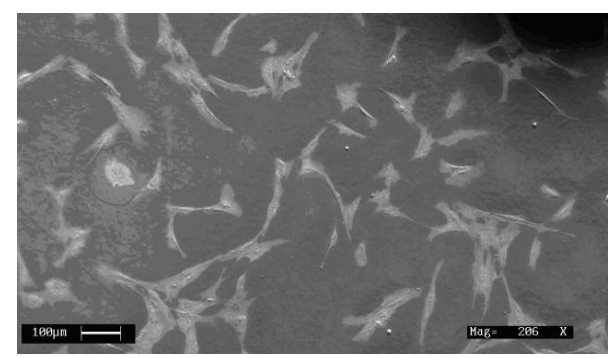

Figure 5 - SEM micrograph of Au coated As-received (AR) sample $24 \mathrm{hrs}$ post seeding.

For the $\mathrm{CO}_{2}$ laser whole area irradiative processing sample CWA26 and sample CWA34 the cells observed were not bipolar in nature. Instead they had a more clumped cell morphology. For example see Figure 7. This indicates that the different fluences used for the $\mathrm{CO}_{2}$ laser whole area irradiative processing lead to modulation in cell signaling compared to other samples.

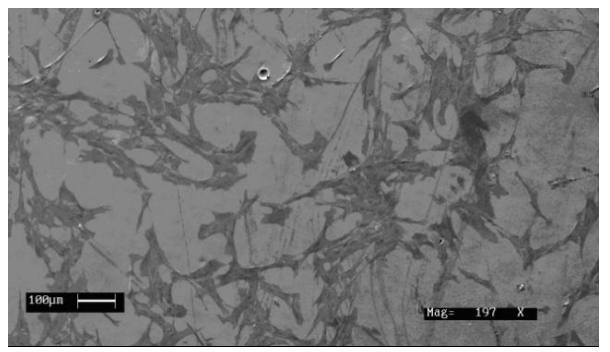

Figure 6 - A typical SEM micrograph of an Au coated $\mathrm{CO}_{2}$ laser surface treated sample (CH100) $24 \mathrm{hrs}$ post seeding.

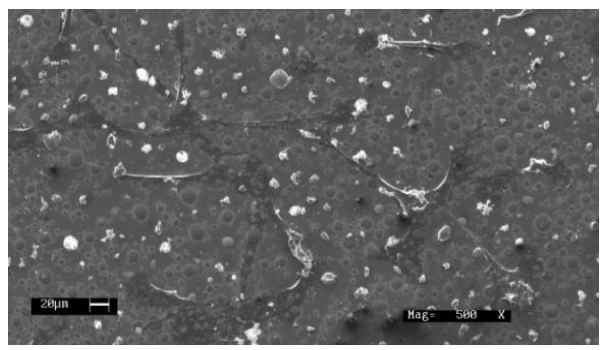

Figure 7 - A typical SEM micrograph of an Au coated $\mathrm{CO}_{2}$ laser whole area irradiated sample (CWA34) 24 hrs post seeding.

In addition, one major difference between the cell growth on the as-received sample (see Figure 5) and the $\mathrm{KrF}$ excimer laser-induced patterned samples is that the excimer patterned nylon 6,6 samples gave rise to some form of directionality (see Figure 8); that is, the cells appeared to be preferentially growing along the grooves formed by the excimer laser. This could have been induced by the surface roughness and surface oxygen content being higher in the grooves produced allowing for preferential cell growth.

Figure 9 shows a typical SEM micrograph for the $\mathrm{KrF}$ excimer laser whole area irradiative processed nylon 6,6 samples after $24 \mathrm{hrs}$ incubation. From this it can be seen that the $\mathrm{KrF}$ excimer whole area irradiative processed samples gave rise to variations in cell morphology in comparison to the as-received sample (see Figure 5) in that, the cells shown in Figure 9 were more clumped radial. This could be attributed to the fact that the combination of differing wettability characteristics and surface oxygen content could have contributed to cell differentiation. 


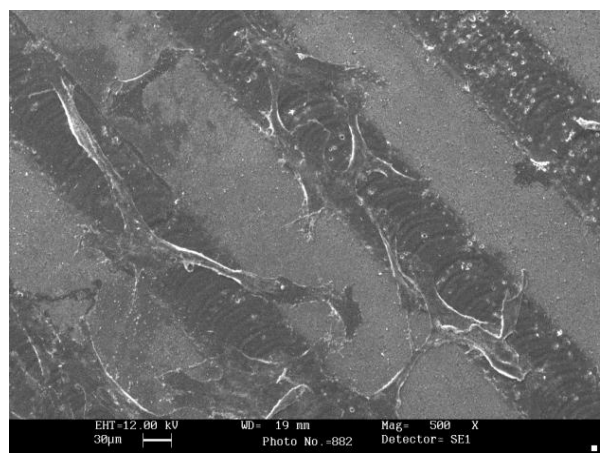

Figure 8 - A typical SEM micrograph of an Au coated $\mathrm{KrF}$ excimer laser-induced patterned sample (ET100) showing an example of cell growth directionality.

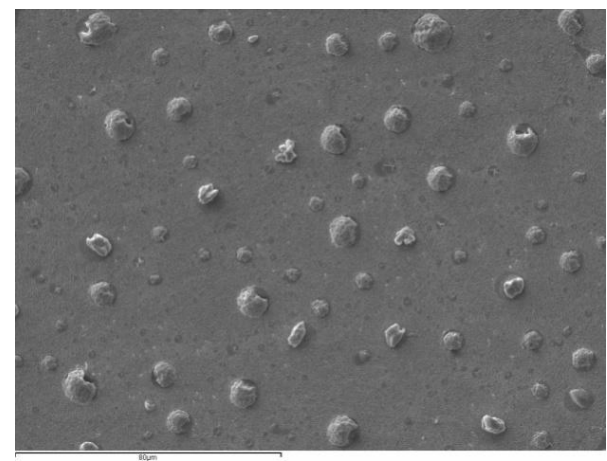

Figure 9 - A typical SEM micrograph of an Au coated $\mathrm{KrF}$ excimer laser whole area irradiated sample (EWA200) 24 hrs post seeding.

\section{Days of Incubation}

With four days of incubation elapsed the cell growth was at an advanced stage for almost all of the samples. From Figure 10 it can be seen that the cell morphology for the as-received sample was radial and coral-like which differed from the $\mathrm{KrF}$ excimer laser-induced patterned samples (see Figure 11) which gave a more clumped like morphology.

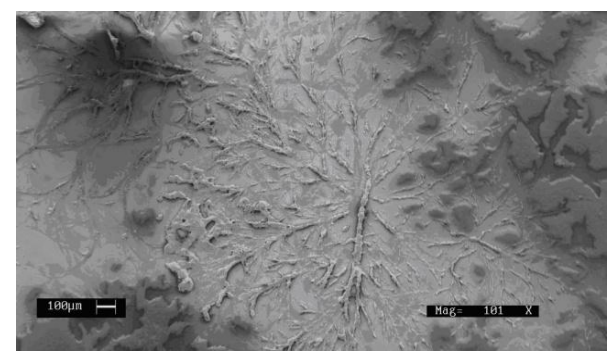

Figure 10 - SEM micrograph of Au coated Asreceived (AR) sample 4days post seeding.

It was seen that the cell morphologies varied somewhat between each sample. For instance, sample EWA100 gave rise to a more clumped like whereas the other samples gave rise to a more radial cell morphology. This, along with the cell differentiation observed (comparing Figures 10,11 and 12) further suggests that the variation in surface properties as a result of the laser treatment had a significant impact upon cell signaling and could be seen as an attractive means for potential use within regenerative medicine.

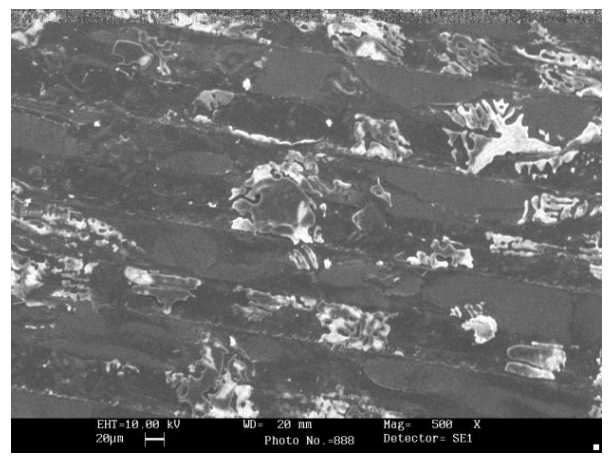

Figure 11 - A typical SEM micrograph of an $\mathrm{Au}$ coated $\mathrm{KrF}$ excimer laser-induced patterned sample (ET50) 4 days post seeding.

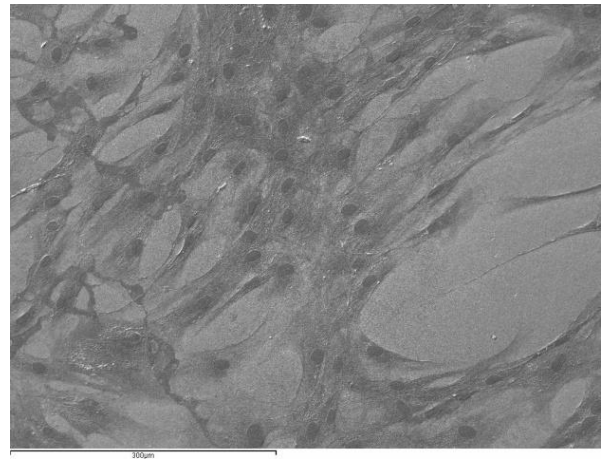

Figure 12 - A typical SEM micrograph of an Au coated $\mathrm{KrF}$ excimer laser whole area irradiated sample (EWA250_1000) $24 \mathrm{hrs}$ post seeding.

\section{Cell Cover Density and Cell Count}

Figure 13 allows one to see that overall the laser surface treated samples did not have a correlative trend between the cell cover density, cell count and $\theta$. This is on account of the laser-induced patterned samples not correlating with the trend observed for the laser whole area irradiative processed samples which can be attributed to the transition in wetting regime. For all laser whole area irradiative processed samples, with the exception of sample CWA102 and sample CWA128, the cell cover density and cell count was an increasing function of $\theta$. It should be noted here that sample CWA102 and sample CWA128 did not follow this trend on the likely account of the toxicity 
dominating the osteoblast cell response arising from the laser treatment.

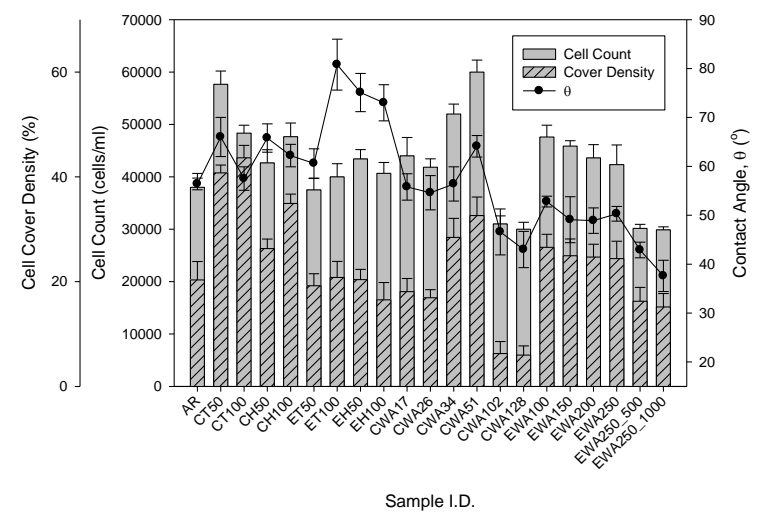

Figure 13 - Histogram showing cell count and cell cover density in relation to $\theta$.

Figure 14 shows that there was no correlative relationship between $\gamma^{\mathrm{P}}, \gamma^{\mathrm{T}}$ and osteoblast cell response for the laser surface treated nylon 6,6 samples when taken as a whole. Similar to $\theta$ (see Figure 13), this can be accounted for by the laser-induced patterned samples which gave rise to somewhat erroneous results on account of the likely transition in wetting regime. The cell cover density, cell count for the entire laser whole area irradiative processed samples can be seen to be an increasing function of $\gamma^{\mathrm{P}}$. However, sample CWA102 and sample CWA128 did not correspond with the trend identified and can be explained by the increase in surface toxicity, hindering cell growth. Leading on, there did not appear to be any correlative relationship between $\gamma^{\mathrm{T}}$ and the osteoblast cell response, indicating that $\gamma^{\mathrm{T}}$ was not a dominant parameter. Even though there was no overall trend observed for $\theta$ and $\gamma^{\mathrm{P}}$ it can still be said within reason that if a surface toxicity was low enough as to not dominate the osteoblast cell response, then it appears likely that $\theta$ and $\gamma^{\mathrm{P}}$ played an important role in determining the osteoblast cell response to the laser whole area irradiative processed nylon 6,6.

It can be seen from Figure 15 that overall, the surface roughness in terms of Ra and Sa did not appear to have a distinct relationship with the osteoblast cell response with regards to cell cover density and cell count. Having said that, for those samples with the largest values of $\mathrm{Sa}$ and $\mathrm{Ra}$ (sample CWA102 and sample CWA128) the surface roughness could be indirectly indicative of the bioactive nature of the $\mathrm{CO}_{2}$ laser whole area irradiative processed nylon 6,6 samples. This is due to the fact that the melting which gave rise to the proposed increase in toxicity which dominated the osteoblast cell response, also gave rise to the significant increase in Ra and Sa.

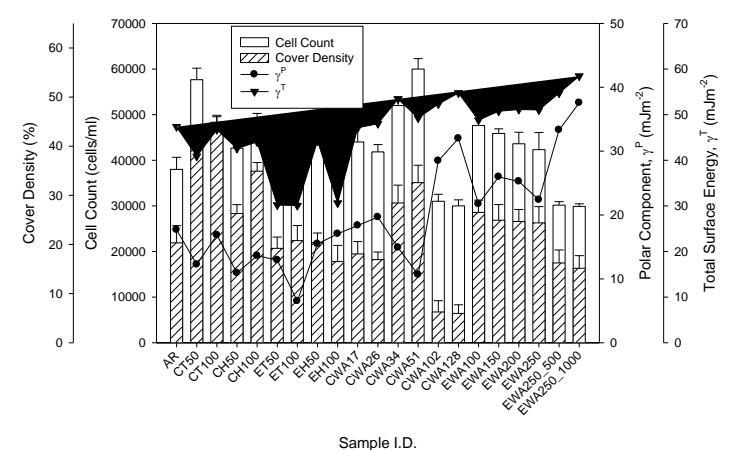

Figure 14 - Histogram showing cell count and cell cover density in relation to $\gamma \mathrm{P}$ and $\gamma \mathrm{T}$.

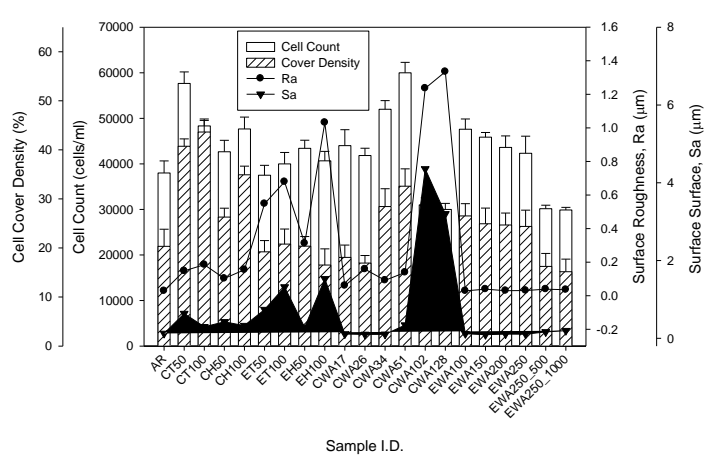

Figure 15 - Histogram showing cell count and cell cover density in relation to $\mathrm{Ra}$ and $\mathrm{Sa}$.

When taking into account all samples with respect to the relationship between the surface oxygen content and osteoblast cell response, it can be seen from Figure 16 that there was no overall trend which could be implemented to predict the osteoblast cell response to the laser surface treated nylon 6,6. Having said that, there was two different observed trends for the laser whole area irradiative processed samples which could potentially be used as an indirect method to estimate whether enhanced osteoblast cell response can be brought about by the nylon 6,6 samples. This is accounted for by the likelihood of the nylon 6,6 samples becoming more toxic upon more laser-induced melting even though the surface oxidized, giving rise to an increase in surface oxygen content.

In terms of cell cover density and cell count it has been seen that below a potential toxicity threshold $\theta$ and $\gamma^{\mathrm{P}}$ dominated the bioactive nature of nylon 6,6 which underwent laser whole area irradiative processing regardless of the laser type used. With this in mind, one can extrapolate that $\theta$ and $\gamma^{\mathrm{P}}$ can be implemented 
to predict how osteoblast cells will react upon laser whole area irradiative processed nylon 6,6.

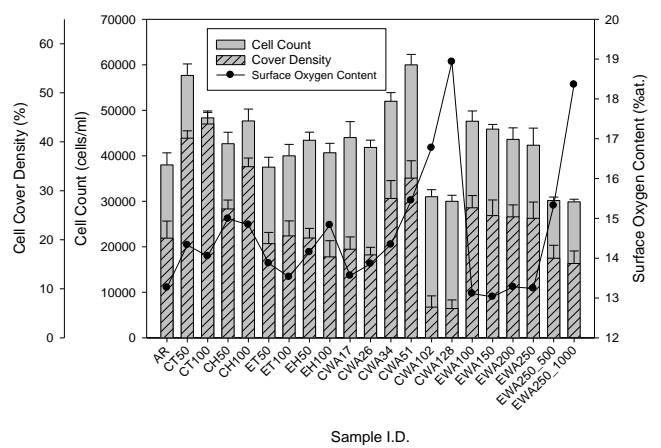

Figure 16 - Histogram showing cell count and cell cover density in relation to surface oxygen content.

\section{Generic Equations Governing Wettability}

It was identified that the laser-induced patterned samples did not correspond with current theory in that an increase in surface roughness did not bring about a reduction in $\theta$ [2]. As a result of this, through analytical analysis a generic equation based on the data for the laser-induced patterns will now be discussed.

One of the equations that arose from the analysis is:

$\theta=114.467-\left(0.694 \gamma^{P}\right)-\left(0.969 \gamma^{T}\right)$

Using the data it was determined that Equation $\{1\}$ had a relatively good fit with Equation $\{1\}$ having an $\mathrm{R}^{2}$ goodness to fit factor of 0.998 , a correlation coefficient of 0.999 and a maximum error of $\pm 1.136^{\circ}$. Figure 16 gives a graph of the predicted contact angles, implementing Equation $\{1\}$, and the actual contact angle recorded. Samples 1 to 9 are those data sets which were used to develop Equation $\{1\}$, samples 10 to 16 is data collected for other laser-induced patterned nylon 6,6 and samples 18 to 21 relates to data collected for laser-induced PMMA [20].

As one can see from Figure 16, Equation $\{1\}$ accurately predicts the contact angle for laser-induced patterned nylon 6,6 (samples 1 to 16). That is, the predicted contact angle was well within the associated error. This implies that Equation $\{1\}$ could be implemented to predict the contact angle for laserpatterned nylon 6,6. By taking the PMMA samples into account (samples 18 to 21) it was found that Equation $\{1\}$ only roughly predicts the correct contact angle. On account of this, it may be necessary to analyse laser-induced patterned PMMA individually as it may require a revised equation based on lasermaterial specific surface modifications.

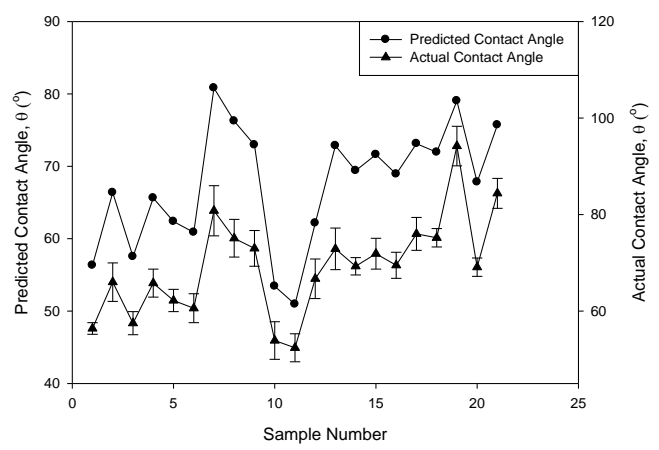

Figure 16-Graph showing the predicted contact angle (using Equation \{1\}) in relation to the actual contact angle for the laser-induced patterned samples.

A more complex equation also arose from the data set which took the form of:

$\theta=112.017+\left(1.445 S a^{2}\right)-\left(0.745 \gamma^{P}\right)-\left(0.893 \gamma^{T}\right)-$ (1.533Sa)

$\{2\}$

Where $\mathrm{Sa}$ is the surface roughness.

Using the data it was determined that Equation $\{2\}$ had a very good fit with Equation $\{2\}$ having an $\mathrm{R}^{2}$ goodness to fit factor of 0.999 , a correlation coefficient of 0.999 and a maximum error of $\pm 0.157^{\circ}$. Figure 17 gives a graph of the predicted contact angles, implementing Equation $\{2\}$, and the actual contact angle recorded. Samples 1 to 9 are those data sets which were used to develop Equation $\{2\}$, samples 10 to 16 is data collected for other laser-induced patterned nylon 6,6 and samples 18 to 21 relates to data collected for laser-induced PMMA [20].

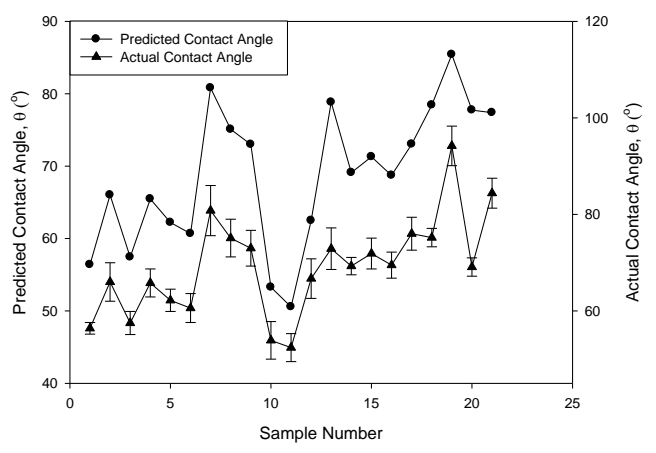

Figure 17 - Graph showing the predicted contact angle (using Equation $\{2\}$ ) in relation to the actual contact angle for the laser-induced patterned samples. 
By taking Figure 17 into consideration one can see that the data used to develop Equation $\{2\}$ had a very good fit with the predicted values. However, when compared with the predicted values in Figure 16, for samples 10 to 21, there was a larger error arising from the predicted values derived from Equation $\{2\}$. On account of this, Equation $\{1\}$ appears to be the more likely candidate for implementation to predict the contact angle for laser-induced patterned nylon 6,6. Having said that, it is necessary to carry out future experiments to confirm the validity of Equation $\{1\}$.

\section{Generic Equations Governing Bioactivity}

Through the trends identified previously it was determined that more fitted correlative trends occurred with the laser whole area irradiated processed samples and the bioactivity observed. This was potentially due to the laser-induced patterned samples giving rise to erroneous results due to the proposed mixed-state wetting regime. As a result of this, only the laser whole area irradiated processed samples were taken into account to develop equations for the cell cover density following $24 \mathrm{hrs}$ and cell count following 4 days of incubation.

By analyzing the cell cover density of the samples one of the equations that arose to determine the cell cover density based on the surface parameters was:

Cell cover density $=0.567+(0.086 S a)+\left(1.76 \gamma^{T} \theta\right)+$ $\left(1.532 \gamma^{P} R a\right)+\left(1.179 \gamma^{T} S O\right)+(0.878 \theta S a)$

Where $\mathrm{Sa}$ is the surface roughness and $\mathrm{SO}$ is the surface oxygen content.

Using the data it was determined that Equation $\{3\}$ had a reasonable fit with Equation $\{3\}$ having an $\mathrm{R}^{2}$ goodness to fit factor of 0.994 , a correlation coefficient of 0.996 and a maximum error of \pm 0.16 . It should be noted that based multi-parameter nature of governing the bioactivity of material it is likely that more results will have to be undertaken to determine and confirm a specific equation to predict the cell cover density of laser whole area processed nylon 6,6. From this point, alternative materials could be tested to determine if the Equation $\{3\}$ could be applied to other materials.

A potential governing equation with regards to cell count after 4 days incubation was found to be:

$$
\begin{aligned}
& \text { Cell count }=0.141+\left(1.878 S O \gamma^{T}\right)-\left(0.446 \gamma^{P} \gamma^{T}\right)- \\
& \left(2.028 S O \gamma^{P}\right) \quad\{4\}
\end{aligned}
$$

Using the data it was determined that Equation $\{4\}$ had a reasonable fit with Equation $\{4\}$ having an $\mathrm{R}^{2}$ goodness to fit factor of 0.970 , a correlation coefficient of 0.985 and a maximum error of \pm 0.27 . Similar to that of Equation $\{3\}$, owed to the multi-parameter nature of this particular type of research more experiments are required to confirm these potential equations.

\section{Conclusions}

Laser surface treatment offers a unique advantage over competing techniques for varying the surface parameters to modulate osteoblast cell response. It has been shown throughout this research that the surface topography and surface chemistry can be modified using laser technology to elicit varying response in terms of wettability characteristics, osteoblast cell cover density and osteoblast cell count.

The laser-induced patterned nylon 6,6 did not correspond to current theory in that an increase in surface roughness should bring about a decrease in the contact angle. Having said that, it was identified that $\theta$ had a strong correlative trend with $\gamma^{\mathrm{P}}$ and $\gamma^{\mathrm{T}}$ for all laser surface-treated nylon 6,6. Following on from this, through analytical analysis, generic equations have been identified and discussed which allow one to accurately predict the contact angle of laser-modified nylon 6,6 using the laser-modified surface energy parameters.

Trends and generic equations for the bioactivity (cell count and cell cover density) of laser whole area irradiative processed nylon 6,6 have also been discussed which have the potential ability to be implemented to predict the biofunctionality of these materials. Whilst trends and equations have been identified it must be noted here that more experiments are necessary to pin down these generic parameters and equations before they can be widely implemented.

Changes in cell morphology over the samples, allowing one to identify a modulation in cell signalling in addition to determining the generic parameters/equations allow one to see the huge potential of laser surface treatment in regenerative medicine. This is due to the fact that this would offer a tool for clinicians to have the ability to predict cell growth and the have the capability of ensuring that certain laser-modified materials can promote modulated biological cell responses.

\section{References}

1. MacGregor K. (2010)The ageing population: U.K. focus for biomedical engineering - policy briefing. The Royal Academy of Engineering.

2. Hao L, Lawrence J. (2005) Laser Surface Treatment of Bio-Implant Materials. John Wiley \& Sons Inc. 
3. Ratner B.D. (2004) Introduction to testing biomaterials, in: Ratner B.D. (ed.) Biomaterials Science. Second Edition. Elsevier Academic Press, p. 355.

4. Ball M.D., Sherlock R., Glynn T. (2004) Cell interactions with laser-modified polymer surfaces. Journal of Materials Science: Materials in Medicine 15 447-449.

5. Roach P., Eglin D., Rohde K., Perry C.C (2007) Modern biomaterials: a review-bulk properties and implications of surface modifications. Journal of Materials Science: Materials in Medicine 18 12631277.

6. Hao L., Lawrence J., Li L. (2005) The wettability modification of bio-grade stainless steel in contact with simulated physiological liquids by the means of laser irradiation. Applied Surface Science 247 453-457.

7. Agathopoulos S., Nikolopoulos P. (1995) Wettability and interfacial interactions in bioceramicbody-liquid systems. Journal of Biomedical Materials Reserach 29 421-429.

8. Teh K.S., Lu Y.W. (2008) Topography and wettability control in biocompatible polymer for BioMEMs applications. Proceedings of the 3rd IEEE Int. Conf. on Nano/Micro Engineered and Molecular Systems, Sanya, China.

9. Botero A.E.C., Mauricio L.T., Luciana M.S.M. (2008) Surface chemistry fundamentals of biosorption of Rhodococcus opacus and its effect in calcite and magnesite flotation. Minerals Engineering 21 83-92.

10. Burton Z, Bhushan B. Hydrophobicity, Adhesion and Friction Properties of Nanopatterned Polymers and Scale Dependence for Micro- and Nanoelectromechanical Systems. Nano Letters 2005;5:1607-1613.

11. Chu P.K. (2007) Plasma surface treatment of artificial orthopedic and cardiovascular biomaterials. Surface and Coatings Technology 201 5601-5606.

12. Lensen M.C., Schulte V.A, Salber J., Diez M., Menges F., Moller M. (2008) Cellular response to novel, micropatterned biomaterials. Pure Applied Chemistry 80 2479-2487.

13. Gollapudi S., Samm K., Klug U., Kling R., Ostendorf A. (2008) Laser controlled multifunctionalization of polymer surfaces for industrial applications. ICALEO 2008 Proceedings 2008; Laser Microprocessing Conference 305-312.
14. Waugh D.G., Lawrence J. (2011) Wettability and osteoblast cell response modulation through UV laser processing of nylon 6,6. Applied Surface Science 257 8798-8812.

15. Waugh D.G., Lawrence J., Morgan D.J., Thomas C.L. (2009) Interaction of $\mathrm{CO}_{2}$ laser-modified nylon with osteoblast cells in relation to wettability. Materials Science and Engineering, Part C 29 25142524.

16. Waugh D.G., Lawrence J. (2008) Wettability characteristics variation of nylon 6,6 by means of $\mathrm{CO}_{2}$ laser generated surface patterns. ICALEO 2008 Proceedings 2008101 61-- 69.

17. Waugh D.G., Lawrence J. (2011) The enhancement of biomimetic apatite coatings by means of $\mathrm{KrF}$ excimer laser surface treatment of nylon 6,6. Lasers in Engineering 21 95-114.

18. Schmidt M., Lipson H. (2009) Distilling Free-Form Natural Laws from Experimental Data. Science 324 81-85.

19. Lee S.M., Kwon T.H. (2007) Effects of intrinsic hydrophobicity on wettability of polymer replicas of a superhydrophobic lotus leaf. Journal of Micromechanics and Microengineering 17687.

20. Waugh D.G., Lawrence J. (2010) On the use of CO2 laser induced patterns to modify the wettability of poly(methyl methamecrylate) (PMMA). Optics and Lasers in Engineering 48 707-715.

\section{Meet the Author}

Dr. David Waugh is a Lecturer in Mechanical Engineering at the University of Lincoln, UK. He forms part of the Lincoln Laser Engineering Research Group in which he primarily focuses on the application of laser technology to life sciences. This involves the laser surface treatment of biomaterials, specifically polymers, to modify the surface topography and surface chemistry to manipulate biological cell growth. 